\title{
The balance of fetuin-A and osteoprotegerin is independently associated with diastolic dysfunction in hemodialysis patients
}

\author{
Ali Talib $^{1}$, Naoki Nakagawa ${ }^{1}$, Erika Saito ${ }^{1}$, Motoki Matsuki ${ }^{1,2}$, Motoi Kobayashi ${ }^{1,3}$, Kazumi Akasaka ${ }^{1}$, \\ Tomoya Hirayama ${ }^{2}$, Hironori Ishida ${ }^{2}$, Nobuyuki Sato ${ }^{1}$ and Naoyuki Hasebe ${ }^{1}$
}

Fetuin-A and osteoprotegerin (OPG) are arterial calcification regulators, which are related to cardiovascular survival in hemodialysis patients. We hypothesized that a balance of these calcification regulators might mediate the progression of left ventricular (LV) diastolic dysfunction in hemodialysis patients. We recruited 63 hemodialysis patients and measured their serum fetuin-A, OPG, arterial stiffness, aortic calcification and echocardiographic parameters, including the transmitral early diastolic velocity/tissue Doppler mitral annular early diastolic velocity ratio $\left(E / E^{\prime}\right)$, and analyzed the relationships between these variables. Fetuin-A levels were significantly and negatively correlated with the ankle-brachial pulse wave velocity (baPWV), aortic calcification score (AOCS), left atrial volume index (LAVI), LV mass index (LVMI) and EIE'. OPG levels and the ratio of OPG to fetuin-A levels were significantly and positively correlated with the baPWV, AOCS, LAVI and EIE. A stepwise multiple regression analysis revealed that $E / E^{\prime}$ was independently correlated with fetuin-A levels $(\beta=-0.334, P=0.02)$, OPG levels $(\beta=0.367$, $P=0.01)$ and the ratio of OPG to fetuin-A $(\beta=0.295, P=0.04)$. Categorizing the patients according to their serum fetuin-A and OPG levels revealed that patients with low fetuin-A and high OPG levels had the highest LAVI, LVMI and EIE values after adjusting for potential confounders. Serum fetuin-A levels negatively reflected, whereas OPG levels and the ratio of OPG to fetuin-A positively reflected an increase in vascular and ventricular stiffness, leading to the aggravation of diastolic dysfunction. Therefore, based on our results, the balance of the tissue calcification regulators fetuin-A and OPG could mediate the progression of LV diastolic dysfunction in hemodialysis patients.

Hypertension Research (2012) 35, 426-433; doi:10.1038/hr.2011.201; published online 1 December 2011

Keywords: diastolic dysfunction; fetuin-A; hemodialysis; osteoprotegerin

\section{INTRODUCTION}

Approximately $40 \%$ of hemodialysis patients have clinical evidence of ischemic heart disease or heart failure. ${ }^{1,2}$ Vascular calcification, which results in increased vascular stiffness, is a common pathological feature of hemodialysis patients ${ }^{1,3}$ and is accompanied by left ventricular hypertrophy (LVH) and diastolic dysfunction. ${ }^{4,5}$ Vascular calcification is an active process controlled by a balance of serum calcification inhibitors and enhancers. Fetuin-A ( $\alpha 2$-Heremans Schmid glycoprotein) is a $62-\mathrm{kDa}$ liver-derived glycoprotein and potent circulatory inhibitor of vascular calcification, which is downregulated by inflammation. ${ }^{6,7}$ Chemically, fetuin-A acts as an inhibitor of spontaneous calcium phosphate precipitation by forming soluble colloidal calciprotein particles. ${ }^{7}$ Fetuin-A-deficient mice experience enhanced myocardial fibrosis and stiffness, ${ }^{8}$ and several studies have documented an inverse relationship between serum fetuin-A levels and survival in dialysis patients. ${ }^{9,10}$
Osteoprotegerin (OPG), a soluble decoy receptor of the osteoclast receptor activator of the nuclear factor $\kappa B$ ligand (RANKL), acts as an important regulator of vascular calcification. ${ }^{11}$ OPG-deficient mice develop severe osteoporosis and vascular calcification. ${ }^{12}$ In contrast, elevated serum levels of OPG are associated with the progression of vascular stiffness and cardiovascular events in hemodialysis patients. 13,14

We therefore hypothesized that an imbalance between fetuin-A and OPG may be associated with increased cardiovascular stiffness and LV diastolic dysfunction in hemodialysis patients.

\section{METHODS}

The eligibility criteria for our study were as follows: inadequacy of dialysis with a urea Kt/V level of $<1.2$, active malignancy, active respiratory infection, acute heart failure, atrial fibrillation at the time of the study, significant valvular heart disease, peripheral arterial disease (ankle-brachial index $<0.9$ ) and chronic use

\footnotetext{
${ }^{1}$ Division of Cardiology, Nephrology, Pulmonology and Neurology, Department of Internal Medicine, Asahikawa Medical University, Asahikawa, Japan; ${ }^{2}$ Department of Nephrology and Urology, Kitasaito Hospital, Asahikawa, Japan and ${ }^{3}$ Department of Cardiology, Hokkaido Prefectural Kitami Hospital, Kitami, Japan

Correspondence: Dr N Nakagawa, Division of Cardiology, Nephrology, Pulmonology and Neurology, Department of Internal Medicine, Asahikawa Medical University, Midorigaoka Higashi 2-1-1-1, Asahikawa 078-8510, Japan.

E-mail: naka-nao@asahikawa-med.ac.jp

Received 13 July 2011; revised 4 September 2011; accepted 26 September 2011; published online 1 December 2011
} 
of glucocorticoids and immunosuppressive agents. We performed echocardiographies in 108 consecutive hemodialysis patients who underwent $4 \mathrm{~h}$ of dialysis three times per week from February 2009 until November 2010 in the dialysis departments of Kitasaito Hospital and Hokkaido Prefectural Kitami Hospital. Among these 108 consecutive patients, 14 with significant valvular heart disease, 12 with peripheral arterial disease, 9 with atrial fibrillation, 6 with inadequacy of dialysis and 4 with acute heart failure were excluded from the study. Finally, 63 hemodialysis patients were recruited for the study. Before entry into the study, written informed consent was obtained from all patients in accordance with the ethical standards laid down in the Declaration of Helsinki.

Before the hemodialysis session, the weight, height and blood pressure (BP) of each patient were measured. Hypertension was defined as a predialysis brachial BP of $>140 / 90 \mathrm{~mm} \mathrm{Hg}$ and/or a current anti-hypertensive treatment. The ankle-brachial pulse wave velocity (baPWV) was measured using a waveform analyzer (VP-1000; Colin Co., Komaki, Japan) after at least 5 min of rest, as previously reported. ${ }^{15}$ The body surface area was calculated according to the following formula: body surface area $=0.6 \times$ height $(\mathrm{m})+0.0128 \times$ weight (kg)-0.1529. Diabetes mellitus was defined based on the World Health Organization criteria. ${ }^{16}$ Fasting predialysis blood samples were taken, and the following laboratory data were measured using standard laboratory methods. To measure serum fetuin-A, OPG and procollagen type III N-terminal amino peptide (PIIIP) levels, the samples were centrifuged immediately, and the serum was stored at $-80^{\circ} \mathrm{C}$ until analysis. Serum fetuin-A and OPG levels were measured using a human fetuin-A enzyme-linked immunosorbent assay kit (Epitope Diagnostics Inc., San Diego, CA, USA) and enzyme-linked immunosorbent assay system (Biomedica, Vienna, Austria). Serum PIIIP levels were measured by immunoradiometry using an IRMA kit (Riagnost P-III-P; CIS Bio International, Ceze, France). All samples were measured in duplicate.

To estimate the aortic calcification score (AOCS), we performed abdominal computed tomography scans with a 3-mm collimation, $5-\mathrm{mm}$ slice thickness and a $35-\mathrm{cm}$ field of view. A standard phantom was used to adjust the scan score brightness, and the calcium levels in a $10-\mathrm{cm}$ segment of the distal abdominal aorta above the aortic bifurcation were used for the analysis. Calcification was defined as a value $>1 \mathrm{~mm}^{2}$ with a density of $>130$ Hounsfield units and was quantified using an Agatston scoring method with an offline software analysis program (Aqualion, Toshiba, Japan), as previously reported. ${ }^{17}$

Before the hemodialysis session, standard echocardiographic measurements were obtained from the left parasternal and apical views according to the recommendations of the American Society of Echocardiography, ${ }^{18}$ using an ALOKA alpha 10 ultrasound system (Aloka, Tokyo, Japan). The LV enddiastolic dimension, $\mathrm{LV}$ end-systolic dimension, interventricular septum thickness and posterior wall thickness were determined using standard echocardiographic 2D measurements. The left atrial volume (LAV) was measured using the biplane method of discs incorporating both apical 4- and 2-chamber views. The LV mass (LVM) was determined using the 2D area-length formula. LAV and LVM were indexed to the body surface area as the LAV index (LAVI) and LVM index (LVMI), respectively. The ejection fraction was calculated by the modified Simpson method. The transmitral inflow was recorded using pulsed wave Doppler recordings at the mitral valve leaflet tips in the apical 3-chamber view. The peak early diastolic filling velocity $(E)$, peak late diastolic filling velocity $(A), E / A$ ratio and deceleration time of the $E$ wave were measured. The tissue Doppler image program was set to the pulsed wave Doppler mode, and sample volumes were positioned at the septal corner of the mitral annulus. ${ }^{19}$ Then, we measured the early diastolic mitral inflow velocity and calculated the ratio

$E$ over $E^{\prime}\left(E / E^{\prime}\right)$ to represent $\mathrm{LV}$ filling pressure. ${ }^{20}$

All values are presented as the mean \pm s.e.m. A univariate linear regression was used for the continuous variables, including the ratio of OPG to fetuin-A. A stepwise multiple regression analysis was performed to determine the correlation and independent variables for $E / E^{\prime}$. We selected the classical cardiovascular risk factors and factors associated with LV diastolic function as the variables. In Model 1, serum fetuin-A levels, age, sex, duration of dialysis, mean $\mathrm{BP}$, heart rate, body mass index, hemoglobin, brain natriuretic peptide level, smoking, medication for hypertension, medication for diabetes, medication for dyslipidemia and prior coronary artery disease (CAD) were included as independent variables with or without the baPWV. In models 2 and 3, serum
OPG levels and the ratio of OPG to fetuin-A, respectively, were included instead of the serum fetuin-A level. Furthermore, we divided the patients into four groups according to the median serum fetuin-A $\left(225 \mathrm{ng} \mathrm{ml}^{-1}\right)$ and OPG $\left(235 \mathrm{ng} \mathrm{ml}^{-1}\right)$ levels as follows: high fetuin-A $\left(\geqslant 225 \mathrm{ng} \mathrm{m}^{-1}\right)$ plus low OPG $\left(\leqslant 234 \mathrm{ng} \mathrm{ml}^{-1}\right)$; high fetuin-A $\left(\geqslant 225 \mathrm{ng} \mathrm{m}^{-1}\right)$ plus high OPG $\left(\geqslant 235 \mathrm{ng} \mathrm{ml}^{-1}\right)$; low fetuin-A $\left(\leqslant 224 \mathrm{ng} \mathrm{ml}^{-1}\right)$ plus low OPG $\left(\leqslant 234 \mathrm{ng} \mathrm{m}^{-1}\right)$; and low fetuin-A $\left(\leqslant 224 \mathrm{ng} \mathrm{ml}^{-1}\right)$ plus high OPG $\left(\geqslant 235 \mathrm{ng} \mathrm{ml}^{-1}\right)$. Their associations with the baPWV, AOCS, LAVI, LVMI and $E / E^{\prime}$ were evaluated with an analysis of covariance for age, sex, duration of dialysis, mean BP, heart rate, body mass index, hemoglobin, brain natriuretic peptide levels, smoking, medication for hypertension, medication for diabetes, medication for dyslipidemia and prior CAD. $P$-values $<0.05$ were considered to be statistically significant. All statistical analyses were performed with the SPSS (Statistical Package for the Social Sciences) software package Version 11.0 for Windows (SPSS Inc., Chicago, IL, USA).

\section{RESULTS}

The clinical parameters of the hemodialysis patients in the present study are summarized in Table 1 . The etiology of renal failure was associated with diabetes mellitus in $47.6 \%$ (30 of 63), hypertensive nephrosclerosis in $25.4 \%$ (16 of 63 ), chronic glomerular disease in $17.4 \%$ ( 11 of 63 ), polycystic kidney disease in $4.7 \%$ ( 3 of 63 ), postnephrectomy due to renal cell carcinoma in $3.2 \%$ (2 of 63 ) and unknown in $1.6 \%$ ( 1 of 63 ) of the patients. A total of 19 patients (30.2\%) had CAD diagnosed by coronary angiography; 3 patients had one vessel disease without intervention, 5 had one vessel disease with a percutaneous coronary intervention, 7 had two vessel diseases with a percutaneous coronary intervention and four had three vessel diseases with coronary artery bypass graft surgery. The $E / E^{\prime}$ was significantly and positively correlated with the baPWV $(r=0.348, P<0.01)$, but not with prior CAD.

As shown in Table 2, the serum fetuin-A levels were inversely related to systolic BP, pulse pressure, serum phosphorus, $\mathrm{Ca} \times \mathrm{P}$ product, LAVI (Figure 1), LVMI (Figure 1), PIIIP levels (Figure 2), E/ $E^{\prime}$ (Figure 2), baPWV and AOCS, and were significantly and positively related to HDL-C, albumin and 25-hydroxyvitamin D levels. A stepwise multiple regression analysis of model 1 revealed that $E / E^{\prime}$ showed an independent correlation only with fetuin-A levels $(\beta$ coefficient $=-0.334$, $P=0.02$ ), suggesting that serum fetuin-A levels were negatively correlated with $E / E^{\prime}$, independent of the traditional risk factors (Table 3 ).

As also shown in Table 2, serum OPG levels were significantly and positively related to the duration of dialysis, plasma human atrial natriuretic peptide, LAVI (Figure 1), PIIIP (Figure 2), E/E' (Figure 2), baPWV and AOCS. There was a positive, but non-significant, correlation with the LVMI (Figure 1). Furthermore, a stepwise multiple regression analysis of model 2 revealed that $E / E^{\prime}$ correlated independently with OPG levels ( $\beta$ coefficient $=0.367, P=0.01$ ), suggesting that OPG levels were positively correlated with $E / E^{\prime}$, independent of the traditional risk factors (Table 3).

We categorized the patients into four groups according to their serum fetuin-A and OPG levels (Group 1: those with high fetuin-A and low OPG, Group 2: those with high fetuin-A and high OPG, Group 3: those with low fetuin-A and low OPG, Group 4: those with low fetuin-A and high OPG) and examined the correlation between each group and the baPWV, AOCS, LAVI, LVMI and $E / E^{\prime}$ after adjusting for potential confounders. We found that the group with low fetuin-A and high OPG levels had the highest baPWV, AOCS (Figure 3), LAVI, LVMI and $E / E^{\prime}$ (all $P<0.05$ for the trend) (Figure 4). Furthermore, the ratio of OPG to fetuin-A was also significantly and positively related to the duration of dialysis, plasma human atrial natriuretic peptide, PIIIP levels, baPWV (Figure 5a), AOCS (Figure 5b), LAVI (Figure 5c), LVMI and $E / E^{\prime}$ (Figure 5d) (all 
Table 1 Characteristics of hemodialysis patients

\begin{tabular}{|c|c|}
\hline$N, \mathrm{M} / \mathrm{F}(\%)$ & $44 / 19(70 / 30)$ \\
\hline Age (years) & $64.8 \pm 1.6$ \\
\hline Duration of dialysis (months) & $94.5 \pm 13.9$ \\
\hline Smokers, $N(\%)$ & $38(60.3 \%)$ \\
\hline Systolic BP (mm Hg) & $143.3 \pm 3.1$ \\
\hline Diastolic BP (mm Hg) & $75.1 \pm 2.0$ \\
\hline Mean BP $(\mathrm{mm} \mathrm{Hg})$ & $96.0 \pm 2.5$ \\
\hline Pulse pressure $(\mathrm{mm} \mathrm{Hg})$ & $68.1 \pm 2.6$ \\
\hline Heart rate $\left(\mathrm{min}^{-1}\right)$ & $73.4 \pm 1.4$ \\
\hline Body mass index $\left(\mathrm{kg} \mathrm{m}^{-2}\right)$ & $21.8 \pm 0.5$ \\
\hline Hypertension, $N(\%)$ & $39(61.9 \%)$ \\
\hline Diabetes mellitus, $N(\%)$ & $30(47.6 \%)$ \\
\hline Coronary artery disease, $N(\%)$ & $19(30.2 \%)$ \\
\hline Hemoglobin $\left(\mathrm{g} \mathrm{dl}^{-1}\right)$ & $10.4 \pm 0.2$ \\
\hline Albumin $\left(\mathrm{g} \mathrm{dl}^{-1}\right)$ & $3.7 \pm 0.1$ \\
\hline Creatinine $\left(\mathrm{mg} \mathrm{dl}^{-1}\right)$ & $8.9 \pm 0.1$ \\
\hline Total cholesterol $\left(\mathrm{mg} \mathrm{dl}^{-1}\right)$ & $151.5 \pm 4.8$ \\
\hline $\mathrm{HDL}-\mathrm{C}\left(\mathrm{mg} \mathrm{dl}^{-1}\right)$ & $44.2 \pm 1.9$ \\
\hline LDL-C (mg dl-1) & $78.8 \pm 3.7$ \\
\hline Calcium $\left(\mathrm{mg} \mathrm{dl}^{-1}\right)$ & $8.9 \pm 0.1$ \\
\hline Phosphorus (mg dl-1) & $5.0 \pm 0$ \\
\hline $\mathrm{Ca} \times \mathrm{P}$ & $44.1 \pm 1$ \\
\hline Intact PTH (pg ml-1) & $144.8 \pm 18$ \\
\hline 25-Hydroxyvitamin $\mathrm{D}\left(\mathrm{pg} \mathrm{ml}^{-1}\right)$ & $11.4 \pm 0.8$ \\
\hline Hemoglobin Alc (\%) & $5.6 \pm 0.2$ \\
\hline C-reactive protein $\left(\mathrm{mg} \mathrm{dl}^{-1}\right)$ & $0.6 \pm 0.2$ \\
\hline hANP $\left(\mathrm{pg} \mathrm{ml}^{-1}\right)$ & $103.9 \pm 12.0$ \\
\hline $\mathrm{BNP}\left(\mathrm{pg} \mathrm{m}^{-1}\right)$ & $308.0 \pm 73.1$ \\
\hline PIIIP (ng ml-1) & $2.4 \pm 0.2$ \\
\hline Fetuin $\mathrm{A}\left(\mathrm{ng} \mathrm{ml}^{-1}\right)$ & $226.8 \pm 6$ \\
\hline Osteoprotegerin (ng ml ${ }^{-1}$ ) & $260.5 \pm 17$ \\
\hline $\operatorname{baPWV}\left(\mathrm{cm} \mathrm{s}^{-1}\right)$ & $2232.0 \pm 95.6$ \\
\hline Ankle-brachial index & $1.03 \pm 0.2$ \\
\hline AOCS (Agatston score) & $10898 \pm 1248$ \\
\hline \multicolumn{2}{|l|}{ Echocardiographic parameters } \\
\hline $\operatorname{LVEF}(\%)$ & $61.1 \pm 1$ \\
\hline Fractional shortening (\%) & $40.1 \pm 6$ \\
\hline IVSd (mm) & $11.4 \pm 0.2$ \\
\hline PWd (mm) & $11.2 \pm 0.2$ \\
\hline LVDd (mm) & $48.5 \pm 0.9$ \\
\hline LVDs (mm) & $32.9 \pm 1.3$ \\
\hline DcT (ms) & $228.1 \pm 8.3$ \\
\hline$E / A$ & $0.80 \pm 0$ \\
\hline$E / E^{\prime}$ & $12.6 \pm 0$ \\
\hline LAVI (ml m-2) & $41.4 \pm 2$ \\
\hline LVMI $\left(\mathrm{g} \mathrm{m}^{-3}\right)$ & $126.0 \pm 5$ \\
\hline \multicolumn{2}{|l|}{ Oral medication, $\mathrm{N}(\%)$} \\
\hline Calcium-channel blockers & $31(49.2)$ \\
\hline ACEI/ARB & $30(47.6)$ \\
\hline Beta blockers & 27 (42.9) \\
\hline Statins & $12(19.1)$ \\
\hline Calcium carbonate & $32(50.8)$ \\
\hline Lanthanum carbonate & $27(42.9)$ \\
\hline $1 \alpha$-Hydroxyvitamin D3 & $34(53.9)$ \\
\hline Sevelamer & $10(15.9)$ \\
\hline Cinacalcet & $4(6.3)$ \\
\hline
\end{tabular}

Abbreviations: $A$, late mitral inflow; ACE, angiotensin-converting enzyme; AOCS, aorta calcification score; ARB, angiotensin II receptor blocker; baPWV, brachial-ankle pulse wave velocity; $\mathrm{BNP}$, brain natriuretic peptide; $\mathrm{BP}$, blood pressure; $\mathrm{DcT}$, deceleration time; $E$, early mitral inflow; $E$, early diastolic mitral annulus motion; hANP, human atrial natriuretic peptide; HDL-C, high-density lipoprotein cholesterol; IVSd, interventricular septum thickness in diastole; LAVI, left atrial volume index; LDL-C; low-density lipoprotein cholesterol; LVDd, left ventricular diameter in diastole; LVDs, left ventricular diameter in systole; LVEF, left ventricular ejection fraction; LVMI, left ventricular volume index; PIIIP, procollagen type III N-terminal amino peptide; PTH, parathyroid hormone; PWd, posterior wall thickness in diastole. peptide; PTH, parathyroid hormone; PWd, posterior wall
Variables are presented as mean \pm s.e.m., or percentage.
Table 2 Correlation of the serum fetuin-A, osteoprotegerin, ratio of the osteoprotegerin to fetuin-A and the clinical parameters

\begin{tabular}{|c|c|c|c|c|c|c|}
\hline \multirow[b]{2}{*}{ Variable } & \multicolumn{2}{|c|}{ Fetuin- $A$} & \multicolumn{2}{|c|}{$O P G$} & \multicolumn{2}{|c|}{ OPG/fetuin A } \\
\hline & $\mathrm{r}$ & $\mathrm{P}$ & $r$ & P & $\mathrm{r}$ & P \\
\hline Age & -0.171 & 0.185 & 0.195 & 0.13 & 0.199 & 0.122 \\
\hline $\operatorname{Sex}(M=1, F=0)$ & -0.097 & 0.455 & 0.138 & 0.115 & 0.143 & 0.269 \\
\hline Duration of dialysis & -0.069 & 0.549 & 0.543 & $<0.001$ & 0.466 & $<0.001$ \\
\hline Systolic BP & -0.425 & 0.001 & 0.078 & 0.58 & 0.145 & 0.299 \\
\hline Mean BP & -0.082 & 0.565 & 0.083 & 0.558 & 0.057 & 0.686 \\
\hline Pulse pressure & -0.535 & 0.001 & 0.187 & 0.179 & 0.291 & 0.035 \\
\hline Body mass index & 0.116 & 0.373 & -0.251 & 0.051 & -0.245 & 0.057 \\
\hline Hemoglobin & 0.169 & 0.169 & -0.05 & 0.705 & -0.097 & 0.463 \\
\hline Albumin & 0.525 & 0.001 & -0.209 & 0.106 & -0.3 & 0.005 \\
\hline Creatinine & -0.018 & 0.888 & -0.041 & 0.752 & -0.023 & 0.858 \\
\hline Total cholesterol & 0.175 & 0.176 & -0.211 & 0.103 & -0.190 & 0.143 \\
\hline HDL-C & 0.271 & 0.033 & 0.161 & 0.212 & -0.201 & 0.118 \\
\hline LDL-C & 0.081 & 0.532 & -0.232 & 0.069 & -0.179 & 0.164 \\
\hline Calcium & -0.074 & 0.572 & 0.121 & 0.353 & 0.085 & 0.514 \\
\hline Phosphorus & -0.325 & 0.01 & 0.193 & 0.133 & 0.243 & 0.057 \\
\hline $\mathrm{Ca} \times \mathrm{P}$ & -0.296 & 0.019 & 0.174 & 0.176 & 0.226 & 0.078 \\
\hline Intact PTH & 0.202 & 0.125 & -0.183 & 0.165 & -0.183 & 0.165 \\
\hline 25-Hydroxyvitamin D & 0.441 & 0.001 & 0.007 & 0.96 & -0.138 & 0.296 \\
\hline C-reactive protein & -0.212 & 0.101 & 0.002 & 0.988 & 0.193 & 0.135 \\
\hline HANP & -0.211 & 0.103 & 0.298 & 0.02 & 0.252 & 0.049 \\
\hline BNP & -0.119 & 0.361 & 0.21 & 0.104 & 0.195 & 0.132 \\
\hline PIIIP & -0.317 & 0.013 & 0.545 & $<0.001$ & 0.589 & $<0.001$ \\
\hline LVEF & 0.237 & 0.237 & -0.055 & 0.68 & -0.175 & 0.188 \\
\hline$E / E$ & -0.343 & 0.007 & 0.345 & 0.007 & 0.334 & 0.009 \\
\hline LAVI & -0.357 & 0.016 & 0.498 & 0.004 & 0.457 & 0.002 \\
\hline LVMI & -0.313 & 0.034 & 0.118 & 0.435 & 0.166 & 0.271 \\
\hline baPWV & -0.277 & 0.029 & 0.622 & $<0.001$ & 0.594 & $<0.001$ \\
\hline AOCS (Agatston score) & -0.362 & 0.004 & 0.257 & 0.046 & 0.326 & 0.010 \\
\hline
\end{tabular}

Abbreviations: AOCS, aorta calcification score; baPWV, brachial-ankle pulse wave velocity; BNP, brain natriuretic peptide; $\mathrm{BP}$, blood pressure; $E$, early mitral inflow; $E^{\prime}$, early diastolic mitral annulus motion; hANP, human atrial natriuretic peptide; HDL-C, high-density lipoprotein cholesterol; LVEF, left ventricular ejection fraction; LAVI, left atrial volume index; LDL-C; lowdensity lipoprotein cholesterol; LVMI, left ventricular volume index; OPG, osteoprotegerin; PIIIP, procollagen type III N-terminal amino peptide; PTH, parathyroid hormone.

$P<0.05)$. A stepwise multiple regression analysis of model 3 revealed that $E / E^{\prime}$ correlated independently with the ratio of OPG to fetuin-A ( $\beta=0.295, P=0.04$ ), thus indicating that the balance of fetuin-A and OPG levels could be useful for identifying increased vascular stiffness and LV diastolic dysfunction in hemodialysis patients.

\section{DISCUSSION}

To the best of our knowledge, this study is the first to describe the circulating serum vascular calcification regulators fetuin-A and OPG, which are closely related to vascular stiffness, and thereby help to elucidate cardiac function in hemodialysis patients. The major findings of our study are that fetuin-A and OPG serum levels are significant modulators for identifying vascular stiffness, ventricular stiffness and diastolic dysfunction in hemodialysis patients.

Vascular stiffness is associated with LV diastolic dysfunction. ${ }^{4}$ Previous reports have demonstrated that marked vascular calcification and stiffness predisposed hemodialysis patients to LVH and cardiac diastolic dysfunction. ${ }^{21}$ We investigated the impact of fetuin-A and OPG serum levels, which are representative of potent vascular calcification regulators, on vascular stiffness and ventricular function. In hemodialysis patients, the levels of serum fetuin-A have been shown to be consistently low, probably due to its enhanced consumption ${ }^{7}$ 

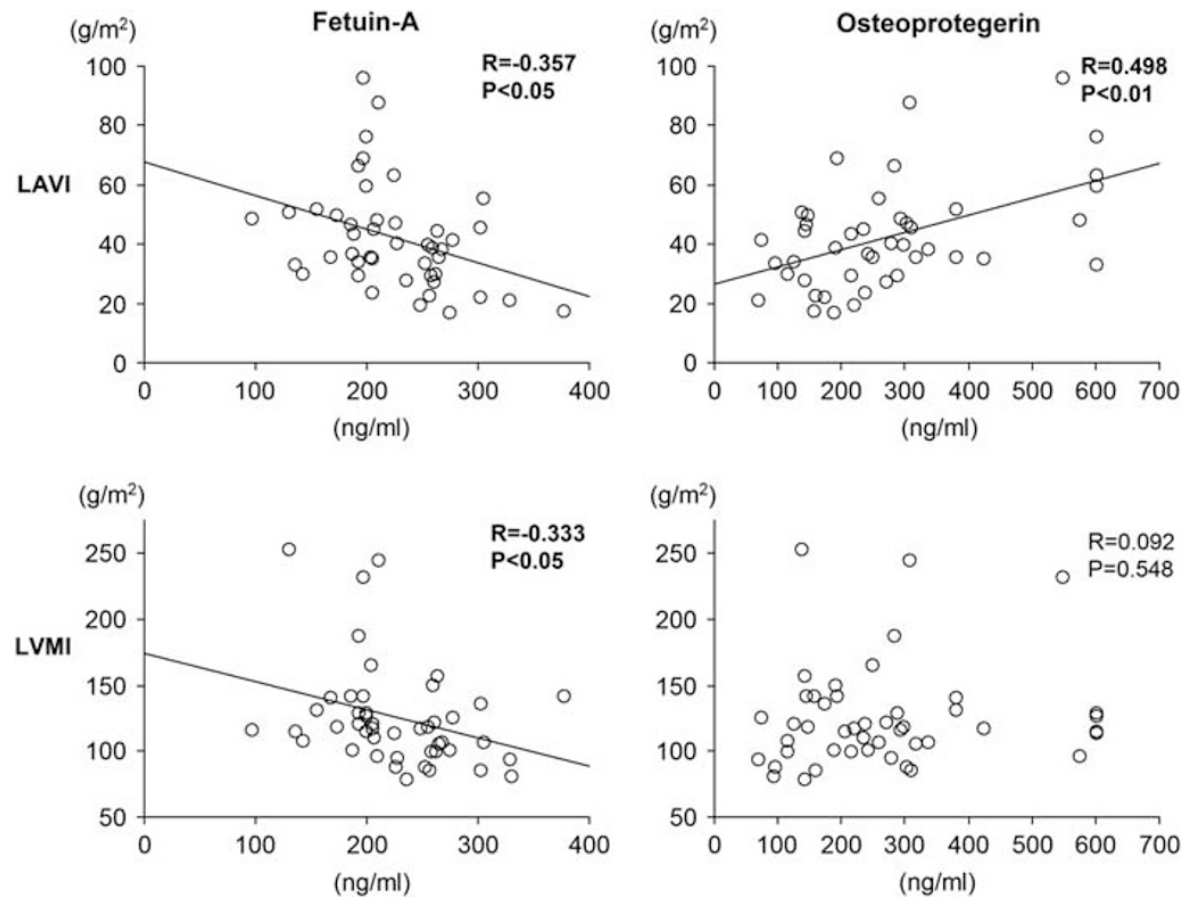

Figure 1 The relationship between fetuin-A levels, osteoprotegerin levels, LAVI and LVMI in all 63 subjects. The LAVI and LVMI correlated significantly and negatively with fetuin-A levels. The correlation between the LVMI and OPG levels was positive, but not significant. LAVI, left atrial volume index; LVMI, left ventricular mass index.
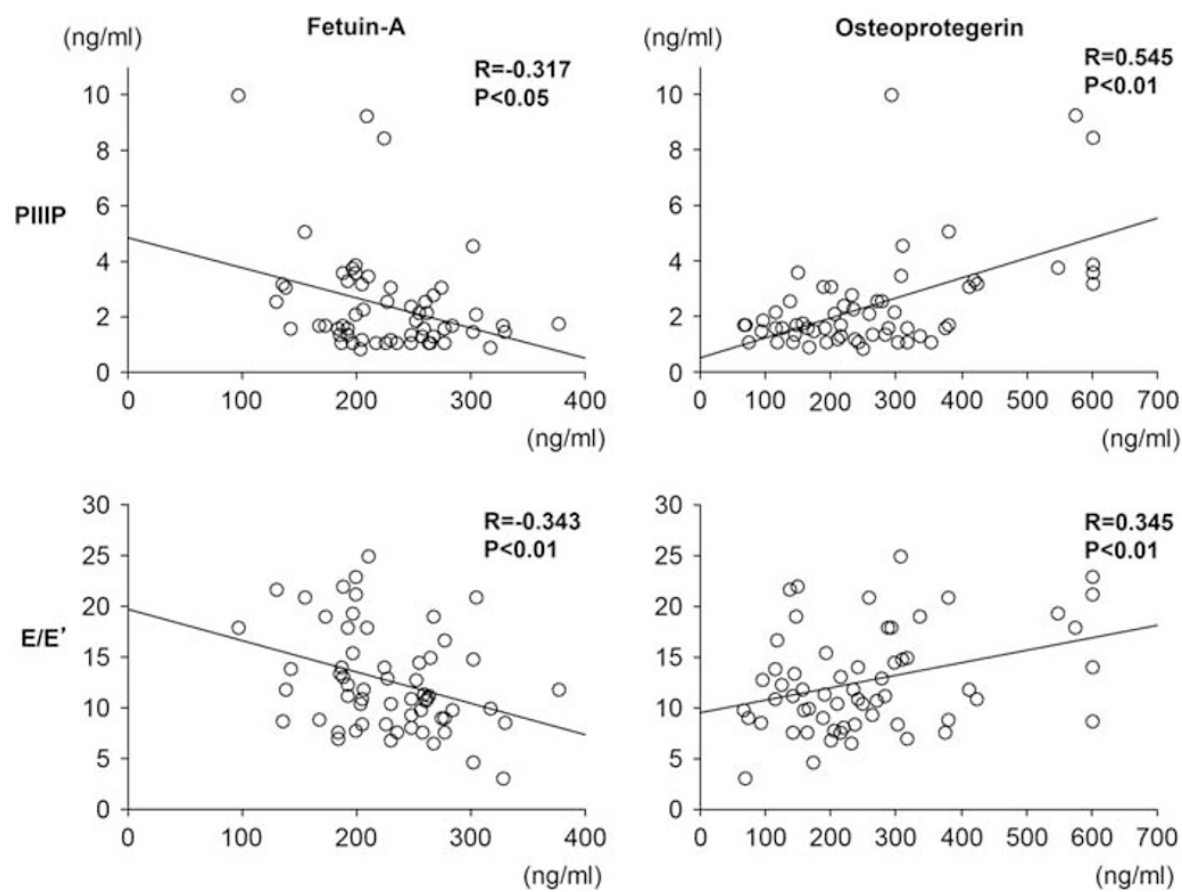

Figure 2 The relationship between fetuin-A levels, osteoprotegerin levels, PIIIP levels and $E / E^{\prime}$ in all 63 subjects. PIIIP levels and $E / E^{\prime}$ correlated significantly and negatively with fetuin-A levels and positively with osteoprotegerin levels. PIIIP, procollagen type III N-terminal amino peptide.

caused by the calcium phosphate load in addition to a chronic microinflammatory status. In our study, the serum fetuin-A levels were inversely related to the AOCS and PWV. Furthermore, we showed for the first time that serum fetuin-A levels were significantly inversely correlated with $E / E^{\prime}$, LAVI and LVMI, thus suggesting that low fetuin-A levels indicate not only an increased vascular stiffness but also an enhanced ventricular stiffness and diastolic dysfunction. 
Table 3 Multiple regression analysis for $E / E^{\prime}$ and related parameters

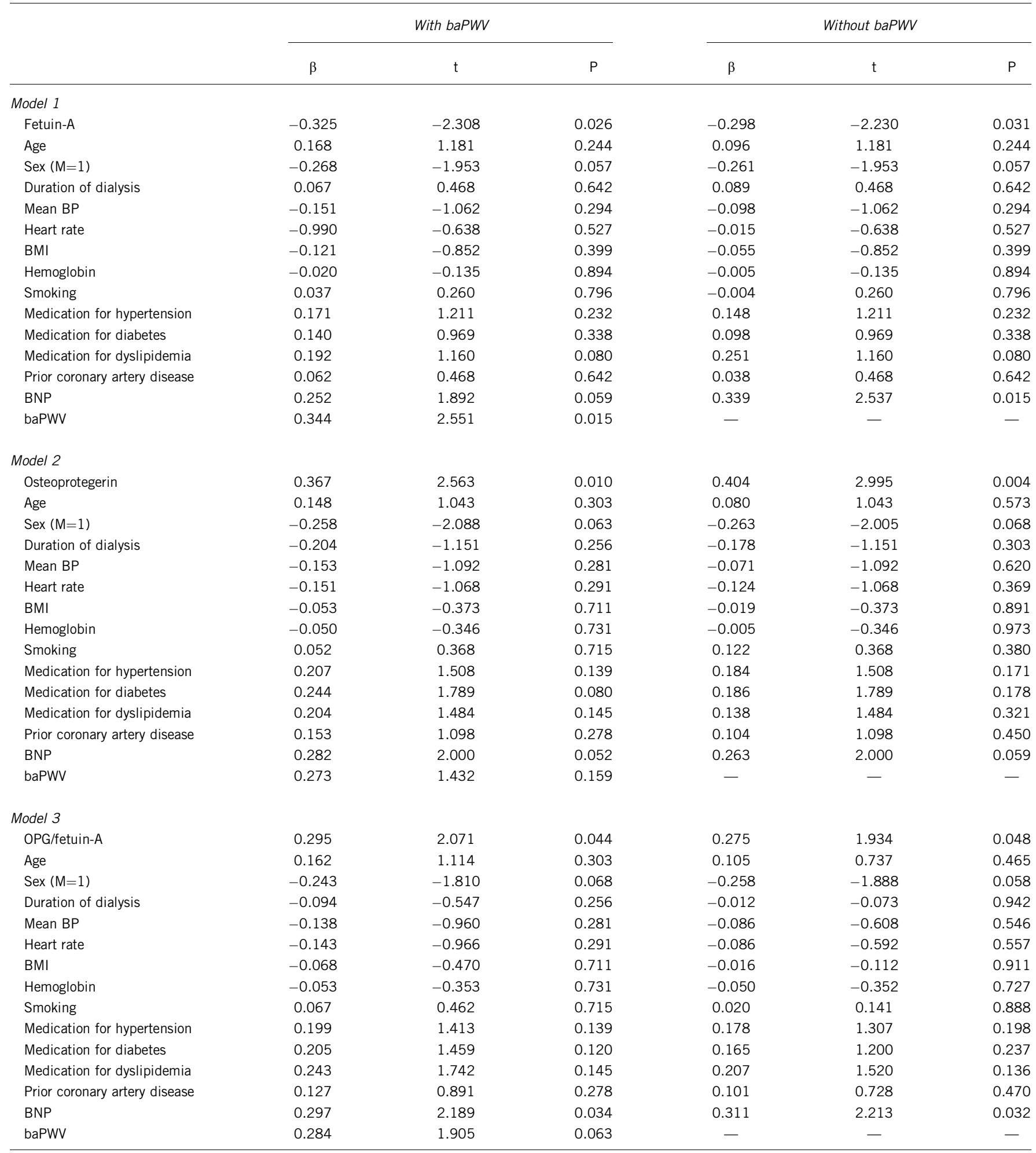

Abbreviations: $\beta, \beta$ coefficient; baPWV, brachial-ankle pulse wave velocity; BMI, body mass index; BNP, brain natriuretic peptide; $\mathrm{BP}$, blood pressure; OPG, osteoprotegerin. Model $1, F$ ratio $=6.243 ; r^{2}=0.221(P=0.004)$ with baPWV, $F$ ratio $=6.203 ; r^{2}=0.220(P=0.004)$ without baPWV.

Model 2, $F$ ratio $=6.571 ; r^{2}=0.219(P=0.004)$ with baPWV, $F$ ratio $=6.176 ; r^{2}=0.210(P=0.008)$ without baPWV.

Model 3, $F$ ratio $=4.780 ; r^{2}=0.178(P=0.013)$ with baPWV, $F$ ratio $=4.782 ; r^{2}=0.188(P=0.010)$ without baPWV.

Because fetuin-A binds to calcium and phosphorus to form a soluble complex (calciprotein particles) ${ }^{7}$ and prevents $\mathrm{Ca} \times \mathrm{PO}_{4}$ precipitation, low serum fetuin-A levels would be expected to predis- pose the patient to vascular calcification and would subsequently lead to increased vascular stiffness, as evidenced by an increase in the PWV. The mechanisms of diastolic dysfunction caused by vascular calcifica- 
a

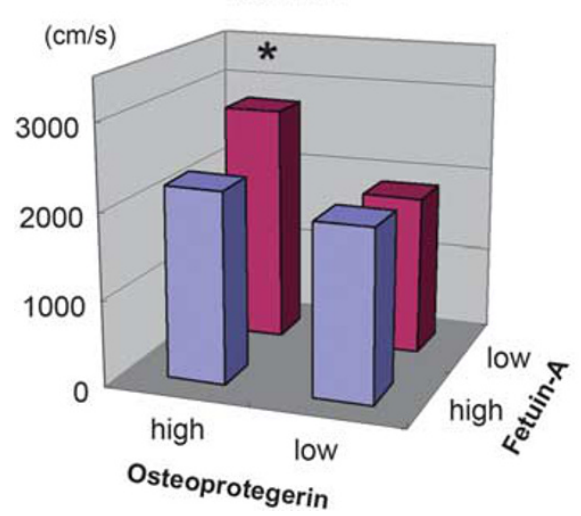

b AOCS

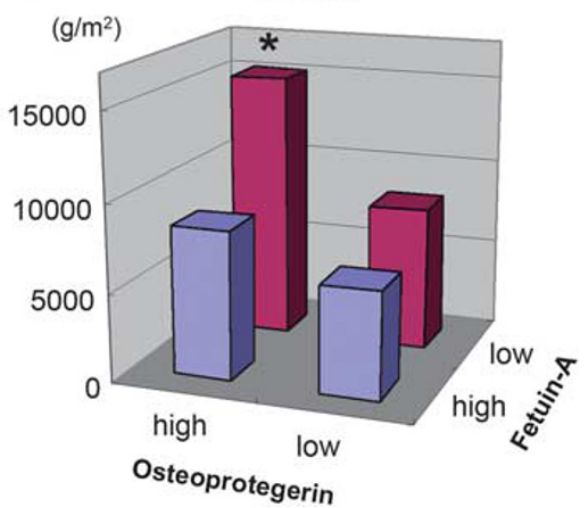

Figure 3 The association between baPWV and AOCS in patients based on the balance between fetuin-A and OPG. After adjusting for age, sex, duration of dialysis, mean BP, heart rate, body mass index, hemoglobin, smoking, medication for hypertension, medication for diabetes, medication for dyslipidemia and prior CAD, significant differences in the baPWV (a) and AOCS (b) were observed across the four groups $(P<0.05)$. The combination of low fetuin-A and high OPG levels was significantly associated with a high baPWV and AOCS. ${ }^{*} P<0.05$ vs. the high fetuin-A and low OPG group. AOCS, aortic calcification score; baPWV, ankle-brachial pulse wave velocity.

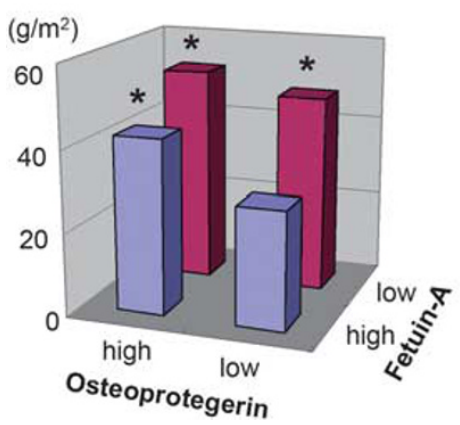

b

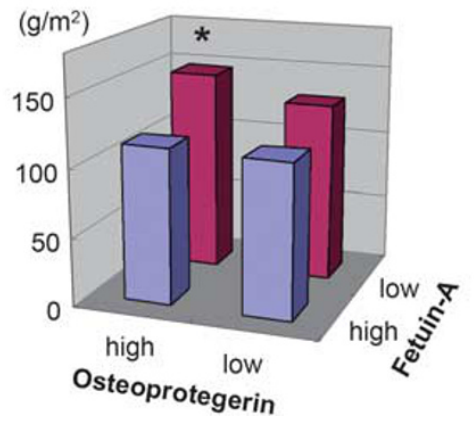

C

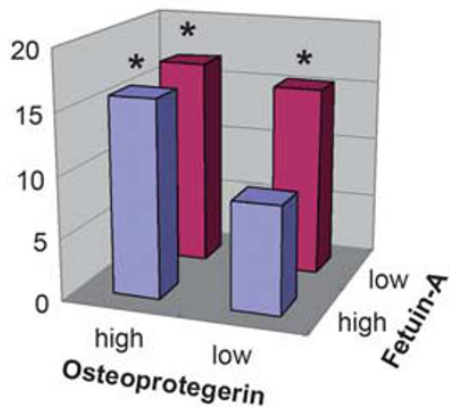

Figure 4 The association of the LAVI, LVMI and E/E' in patients based on a combination of fetuin-A and OPG. After adjusting for age, sex, duration of dialysis, mean BP, heart rate, body mass index, hemoglobin, smoking, medication for hypertension, medication for diabetes, medication for dyslipidemia and prior CAD, significant differences in the LAVI (a), LVMI (b), and $E / E^{\prime}$ (c) were observed across the four groups $(P<0.05)$. The combination of low fetuin-A and high OPG levels was significantly associated with a high LAVI, LVMI and $E / E$. ${ }^{*} P<0.05$ vs. the high fetuin-A and low OPG group.

tion may be ascribed to the earlier arrival of the reflected wave during end systole in stiff and heavily calcified arteries. The reflected wave during end systole would thus merge with the incident wave, which is generated by the ventricular systolic ejection, leading to higher systolic pressures and lower diastolic pressures. ${ }^{22}$ The resultant increase in the afterload during LV systole and reduction in the coronary perfusion pressure during LV diastole may lead to LV hypertrophy and slowing of the LV relaxation. ${ }^{23,24}$ However, hyperphosphatemia in uremic animal models has also been found to aggravate myocardial fibrosis and cause $\mathrm{LVH}^{8}$ As a chelating agent of calcium phosphate, fetuin-A might protect the myocardium against the harmful effects of hyperphosphatemia. Furthermore, fetuin-A also acts as a soluble receptor-like antagonist of TGF- $\beta,{ }^{7}$ resulting in persistent low serum fetuin-A levels, which might directly contribute to increased LV stiffness and hypertrophy. Our novel finding that a significant inverse correlation exists between levels of fetuin-A and PIIIP, which is a marker of myocardial fibrosis, also supports this mechanism.

We found a significant positive relationship between OPG levels and AOCS, as well as the PWV, as previously reported. OPG could act as a survival factor for serum-deprived smooth muscle cells. ${ }^{11}$ Hence, high OPG levels in patients with a high AOCS may reflect an incomplete defense mechanism against factors that promote heavy arterial calcification, ${ }^{25}$ which ultimately results in increased ventricular stiffness, as explained above.

Another new finding in this study was the significant positive correlation between OPG levels, plasma PIIIP levels and LV E/E', which reflects an increased LV stiffness and aggravated diastolic dysfunction, as was observed in the hemodialysis patients. Ueland et al. ${ }^{26}$ showed increased expression of OPG, RANK and RANKL in the failed human myocardium, and they hypothesized that high OPG expression levels could be protective against the harmful effects of RANKL on the myocardium in patients with heart failure, thus suggesting that OPG has a cardiac-protective role. However, another possibility should also be considered. Namely, high serum OPG levels have been associated with LVH in hypertensive African-Americans. ${ }^{27}$ OPG also induces an anti-apoptotic effect by binding to tumor necrosis factor-related apoptosis-inducing ligands, which reduces the number of apoptotic bodies. ${ }^{11}$ Although we could not demonstrate a significant positive relationship between 

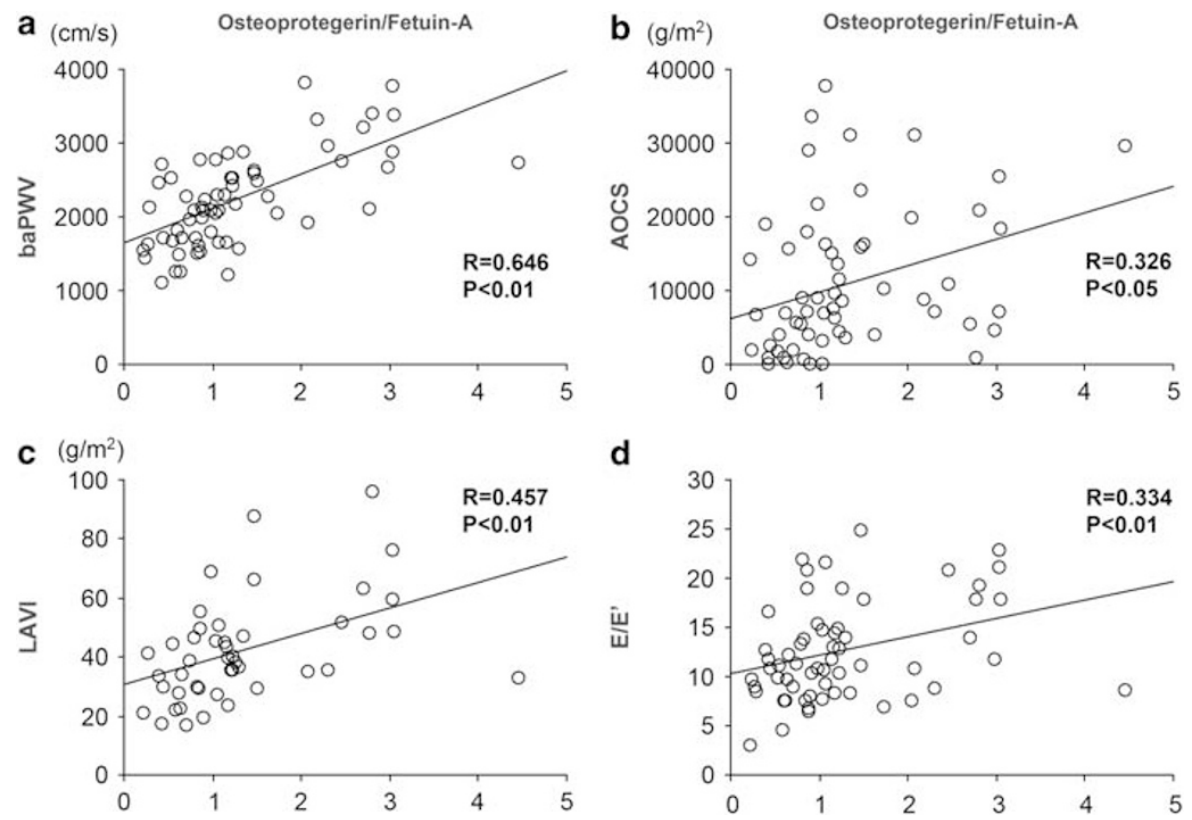

Figure 5 The relationship between the ratio of OPG to fetuin-A, baPWV (a), AOCS (b), LAVI (c) and EIE' (d) in all 63 subjects. The ratio of OPG to fetuin-A correlated significantly and positively with the baPWV, AOCS, LAVI and E/E'. A full color version of this figure is available at the Hypertension Research journal online.

OPG levels and LVMI in the present study, a significant positive relationship between OPG and PIIIP levels, and between OPG levels and the LV diastolic dysfunction parameter may indicate that OPG has a potential role in promoting diastolic dysfunction in hemodialysis patients.

When we categorized the hemodialysis patients according to the median level of serum fetuin-A levels, which served as a negative marker of diastolic dysfunction, and serum OPG levels, which served as a positive marker of diastolic dysfunction, we found that low serum fetuin-A and high serum OPG levels were present in patients with the worst spectrum of LA enlargement, LVH and diastolic dysfunction. The combination of low fetuin-A and high OPG is a significant biomarker of LV diastolic dysfunction, thereby potentially indicating a high risk of complications with atrial fibrillation in hemodialysis patients. Hence, measuring the serum levels of both markers and the ratio of OPG to fetuin-A could be useful for identifying patients with imminent diastolic heart failure. Furthermore, the ratio of OPG to fetuin-A remained significant after adjusting for vascular stiffness in Table 3, suggesting that fetuin-A and OPG might be associated with the progression of LV diastolic dysfunction directly and indirectly through an increase in the afterload owing to elevating vascular stiffness.

This study has several limitations. First, this study sampled a relatively small size of the population and used a cross-sectional design. Second, we might not have completely excluded asymptomatic CAD because we diagnosed CAD by coronary angiography. Third, peripheral arterial disease is commonly observed in hemodialysis patients, but we excluded those patients defined by an ankle-brachial index of $<0.9$ because of the lack of baPWV measurements. Thus, the subgroup of patients included in this study might not be representative of hemodialysis patients as a whole because of the restrictive eligibility criteria. Larger prospective studies will therefore be needed to confirm our results.

In conclusion, a balance of the tissue calcification regulators fetuinA and OPG might mediate the progression of LV diastolic dysfunction by increasing the afterload by elevating vascular stiffness in hemodialysis patients.

\section{CONFLICT OF INTEREST}

The authors declare no conflict of interest.

\section{ACKNOWLEDGEMENTS}

We thank Kozue Kanzaki and the staff of Kitasaito Hospital and Hokkaido Prefectural Kitami Hospital for their help in data collection and interpreting the echocardiographs.

1 Sarnak MJ, Levey AS, Schoolwerth AC, Coresh J, Culleton B, Hamm LL, McCullough PA, Kasiske BL, Kelepouris E, Klag MJ, Parfrey P, Pfeffer M, Raij L, Spinosa DJ, Wilson PW. Kidney disease as a risk factor for development of cardiovascular disease - A statement from the American Heart Association councils on kidney in cardiovascular disease, high blood pressure research, clinical cardiology, and epidemiology and prevention. Circulation 2003; 108: 2154-2169.

2 Shastri S, Samak MJ. Cardiovascular disease and CKD: core curriculum 2010. Am J Kidney Dis 2010; 56: 399-417.

3 Zapolski T. Malnutrition-inflammation complex syndrome: link between end-stage renal disease, atherosclerosis and valvular calcification. Hypertens Res 2010; 33: 541-543.

4 Sigrist M, Bungay P, Taal MW, Mclntyre CW. Vascular calcification and cardiovascular function in chronic kidney disease. Nephrol Dial Transplant 2006; 21: 707-714.

5 Kass DA. Ventricular arterial stiffening: integrating the pathophysiology. Hypertension 2005; 46: 185-193.

6 Schafer C, Heiss A, Schwarz A, Westenfeld R, Ketteler M, Floege J, Muller-Esterl W, Schinke T, Jahnen-Dechent W. The serum protein alpha(2)-Heremans-Schmid glycoprotein/fetuin-A is a systemically acting inhibitor of ectopic calcification. J Clin Invest 2003; 112: 357-366.

7 Jahnen-Dechent W, Schafer C, Ketteler M, McKee MD. Mineral chaperones: a role for fetuin-A and osteopontin in the inhibition and regression of pathologic calcification. J Mol Med 2008; 86: 379-389.

8 Merx MW, Schafer C, Westenfeld R, Brandenburg V, Hidajat S, Weber C, Ketteler M, Jahnen-Dechent W. Myocardial stiffness, cardiac remodeling, and diastolic dysfunction in calcification-prone fetuin-A-deficient mice. J Am Soc Nephrol 2005; 16: 3357-3364.

9 Ketteler M, Bongartz P, Westenfeld R, Wildberger JE, Mahnken AH, Bohm R, Metzger T, Wanner C, Jahnen-Dechent W, Floege J. Association of low fetuin-A (AHSG) concentra- 
tions in serum with cardiovascular mortality in patients on dialysis: a cross-sectional study. Lancet 2003; 361: 827-833.

10 Wang AY, Woo J, Lam CW, Wang M, Chan IH, Gao P, Lui SF, Li PK, Sanderson JE. Associations of serum fetuin-A with malnutrition, inflammation, atherosclerosis and valvular calcification syndrome and outcome in peritoneal dialysis patients. Nephrol Dial Transplant 2005; 20: 1676-1685.

11 Van Campenhout A, Golledge J. Osteoprotegerin, vascular calcification and atherosclerosis. Atherosclerosis 2009; 204: 321-329.

12 Bucay N, Sarosi I, Dunstan CR, Morony S, Tarpley J, Capparelli C, Scully S, Tan HL, Xu W, Lacey DL, Boyle WJ, Simonet WS. osteoprotegerin-deficient mice develop early onset osteoporosis and arterial calcification. Genes Dev 1998; 12 : 1260-1268.

13 Speer G, Fekete BC, EI Hadj Othmane T, Szabo T, Egresits J, Fodor E, Kiss I, Logan AG, Nemcsik J, Szabo A, Nemeth ZK, Szathmari M, Tisler A. Serum osteoprotegerin level, carotid-femoral pulse wave velocity and cardiovascular survival in haemodialysis patients. Nephrol Dial Transplant 2008; 23: 3256-3262.

14 Nishiura R, Fujimoto S, Sato Y, Yamada K, Hisanaga S, Hara S, Nakao H, Kitamura K. Elevated osteoprotegerin levels predict cardiovascular events in new hemodialysis patients. Am J Nephrol 2009; 29: 257-263.

15 Nakagawa N, Takahashi F, Chinda J, Kobayashi M, Hayashi Y, Abe M, Saijo $Y$ Kikuchi K, Hasebe N. A newly estimated glomerular filtration rate is independently associated with arterial stiffness in Japanese patients. Hypertens Res 2008; 31: 193-201.

16 Alberti KG, Zimmet PZ. Definition, diagnosis and classification of diabetes mellitus and its complications. Part 1: diagnosis and classification of diabetes mellitus provisional report of a WHO consultation. Diabet Med 1998; 15: 539-553.

17 Wong ND, Gransar H, Shaw L, Polk D, Moon JH, Miranda-Peats R, Hayes SW, Thomson LE, Rozanski A, Friedman JD, Berman DS. Thoracic aortic calcium versus coronary artery calcium for the prediction of coronary heart disease and cardiovascular disease events. JACC Cardiovasc Imaging 2009; 2: 319-326.

18 Lang RM, Bierig M, Devereux RB, Flachskampf FA, Foster E, Pellikka PA, Picard MH, Roman MJ, Seward J, Shanewise JS, Solomon SD, Spencer KT, Sutton MS, Stewart WJ. Recommendations for chamber quantification: a report from the American Society of Echocardiography's Guidelines and Standards Committee and the Chamber Quantifica- tion Writing Group, developed in conjunction with the European Association of Echocardiography, a branch of the European Society of Cardiology. J Am Soc Echocardiogr 2005; 18: 1440-1463.

19 Ommen SR, Nishimura RA, Appleton CP, Miller FA, Oh JK, Redfield MM, Tajik AJ. Clinical utility of Doppler echocardiography and tissue Doppler imaging in the estimation of left ventricular filling pressures: a comparative simultaneous Dopplercatheterization study. Circulation 2000; 102: 1788-1794.

20 Sohn DW, Kim YJ, Park YB, Choi YS. Clinical validity of measuring time difference between onset of mitral inflow and onset of early diastolic mitral annulus velocity in the evaluation of left ventricular diastolic function. J Am Coll Cardiol 2004; 43 . 2097-2101.

21 Fujiu A, Cigawa T, Matsuda N, Ando Y, Nitta K. Aortic arch calcification and arterial stiffness are independent factors for diastolic left ventricular dysfunction in chronic hemodialysis patients. Circ J 2008; 72: 1768-1772.

22 Laurent S, Cockcroft J, Van Bortel L, Boutouyrie P, Giannattasio C, Hayoz D, Pannier B, Vlachopoulos C, Wilkinson I, Struijker-Boudier H. Expert consensus document on arterial stiffness: methodological issues and clinical applications. Eur Heart J 2006; 27: 2588-2605.

23 Edwards NC, Ferro CJ, Townend JN, Steeds RP. Aortic distensibility and arterialventricular coupling in early chronic kidney disease: a pattern resembling heart failure with preserved ejection fraction. Heart 2008; 94: 1038-1043.

24 Takeuchi T, Ishii Y, Kikuchi K, Hasebe N. Ischemic preconditioning effect of prodromal angina is attenuated in acute myocardial infarction patients with hypertensive left ventricular hypertrophy. Circ J 2011; 75: 1192-1199.

25 Anand DV, Lahiri A, Lim E, Hopkins D, Corder R. The relationship between plasma osteoprotegerin levels and coronary artery calcification in uncomplicated type 2 diabetic subjects. J Am Coll Cardiol 2006; 47: 1850-1857.

26 Ueland T, Yndestad A, Oie E, Florholmen G, Halvorsen B, Froland SS, Simonsen S, Christensen G, Gullestad L, Aukrust P. Dysregulated osteoprotegerin/RANK ligand/RANK axis in clinical and experimental heart failure. Circulation 2005; 111 : 2461-2468.

27 Noheria A, Mosley Jr TH, Kullo IJ. Association of serum osteoprotegerin with left ventricular mass in African American adults with hypertension. Am J Hypertens 2010; 23: 767-774. 